Trans

continentales
Transcontinentales

Sociétés, idéologies, système mondial

$10 / 11 \mid 2011$

La ruée vers la terre

\title{
Le « production grabbing » et la transnationalisation de l'agriculture (sud-) africaine
}

The "grabbing production" and the transnationalization of (South) african

Agriculture

\section{Antoine Ducastel et Ward Anseeuw}

\section{OpenEdition}

\section{Journals}

Édition électronique

URL : http://journals.openedition.org/transcontinentales/1080

DOI : 10.4000/transcontinentales. 1080

ISBN : 978-2-7351-1572-3

ISSN : 1775-397X

Éditeur

Editions de la maison des sciences de l'homme

Référence électronique

Antoine Ducastel et Ward Anseeuw, «Le «production grabbing » et la transnationalisation de l'agriculture (sud-) africaine », Transcontinentales [En ligne], 10/11 | 2011, document 3, mis en ligne le 19 octobre 2011, consulté le 07 septembre 2020. URL : http://journals.openedition.org/ transcontinentales/1080 ; DOI : https://doi.org/10.4000/transcontinentales.1080

Ce document a été généré automatiquement le 7 septembre 2020.

Tous droits réservés 


\section{Le « production grabbing » et la transnationalisation de l'agriculture (sud-) africaine}

The "grabbing production" and the transnationalization of (South) african

Agriculture

Antoine Ducastel et Ward Anseeuw

1 Au cours des dernières années, on a assisté à une "redécouverte » de l'agriculture en tant que domaine d'activité stratégique. Ce secteur s'était vu progressivement relégué au second plan, à la fois dans les agendas de politiques publiques pour qui les mythes d'une croissance essentiellement urbaine et le faible coût des produits agricoles contribuaient à entretenir ce désintérêt chronique, et dans les stratégies des investisseurs privés qui étaient découragés par les faibles marges financières disponibles et les risques inhérents à cette activité ${ }^{1}$.

2 En 2008, la crise alimentaire a suscité un nouvel intérêt de ces acteurs pour l'agriculture. D'une part, même si les promesses tardent à se matérialiser et qu'il ne s'agit, peut-être, que d'une "révolution» discursive, les autorités nationales et internationales reconnaissent désormais l'urgence de la situation agricole dans les pays du Sud. Ainsi, depuis 2008, huit pays africains ont accru leurs contributions au développement agricole jusqu'à $10 \%$ de leur budget national ${ }^{2}$. De plus, en 2009, au sommet d'Aquila, 20 milliards de dollars ont été promis par les membres du G8 pour lutter contre la faim ${ }^{3}$. D'autre part, cette crise a également entraîné un réveil du secteur privé. En effet, elle a fait tomber le mythe d'un faible prix permanent des denrées alimentaires. Désormais, ce secteur présente des perspectives de retombées financières beaucoup plus intéressantes. De plus, certaines évolutions structurelles (augmentation de la population, pression sur les ressources naturelles, changement des régimes alimentaires, etc.) renforcent cette perception d'un secteur agricole porteur à moyen terme $e^{4}$. 
3 Ainsi, l'agriculture africaine se caractérise aujourd'hui, et pour la première fois depuis les années 1980, par une hausse significative des investissements nationaux mais, surtout, internationaux. Paul Runge, le directeur de l'« Africa Project Access », note ainsi que «le nombre de projets d'investissement dans le secteur agricole en Afrique subsaharienne est sans précédent $»^{5}$. On observe en effet une multiplication des projets d'investissements étrangers sur le continent africain et un accroissement des investissements directs étrangers (IDE) à destination de l'agriculture. En 2008, les IDE en direction du continent africain atteignaient 87,6 milliards de dollars (soit $27 \%$ de plus que l'année précédente), dont un tiers étaient dirigés vers les industries extractives et agricoles dans les pays d'Afrique subsaharienne (soit 27 milliards de dollars) ${ }^{6}$.

4 À propos de la nature de ces projets, on voit apparaître des investissements portant directement sur les ressources naturelles, et notamment la terre. Cette catégorie, souvent qualifiée de «land grabbing», voit des investisseurs, publics ou privés, nationaux ou étrangers, acquérir des terres à vocation agricole. Cette question fait actuellement l'objet d'un large traitement scientifique ${ }^{7}$. Cependant, il semble que ce phénomène ne représente que la partie émergée de dynamiques plus larges. En effet, le phénomène d'acquisition foncière cacherait des dynamiques de contrôle direct de la production non seulement par l'acquisition des terres, mais aussi par le contrôle sur un ou des segments de la chaîne de production (" production grabbing »).

5 Moins visible, ce production grabbing est moins analysé. Il est pourtant générateur de transformations au niveau local notamment sur les structures de production et au niveau international en termes de mode d'action et de paradigme du développement. Afin de mieux comprendre ces évolutions, cet article détaille plusieurs modèles qui ont été développés en Afrique du Sud. Ce pays connaît, en effet, une restructuration rapide dans ce sens. À ce titre, mais également du fait de sa position géostratégique, il représente un exemple-modèle pour les autres pays en cours de transformation agricole.

6 La deuxième section ci-après donne une perspective plus globale de ces investisseurs agricoles et $\mathrm{du}$ vecteur par lequel s'effectuent les restructurations actuelles. Se concentrant sur les catégories nouvelles, celles des banques, des entreprises d'ingénierie agricole et des fonds d'investissement, la troisième section est consacrée à la présentation de nouveaux modèles de production. Enfin, la quatrième soulève des réflexions liées à l'internationalisation de l'agriculture et aux changements structurels des économies agricoles en Afrique.

7 Ces flux d'investissements reflètent une internationalisation et une transnationalisation du secteur agricole dans son ensemble. En effet, à côté des investissements réalisés par les pays occidentaux, on voit désormais apparaître des flux Sud-Sud, en provenance de l'Asie (Chine, Corée, Inde), du Moyen-Orient (Arabie saoudite) ou de l'Afrique (Afrique du Sud) (Figure 1). 
Figure 1

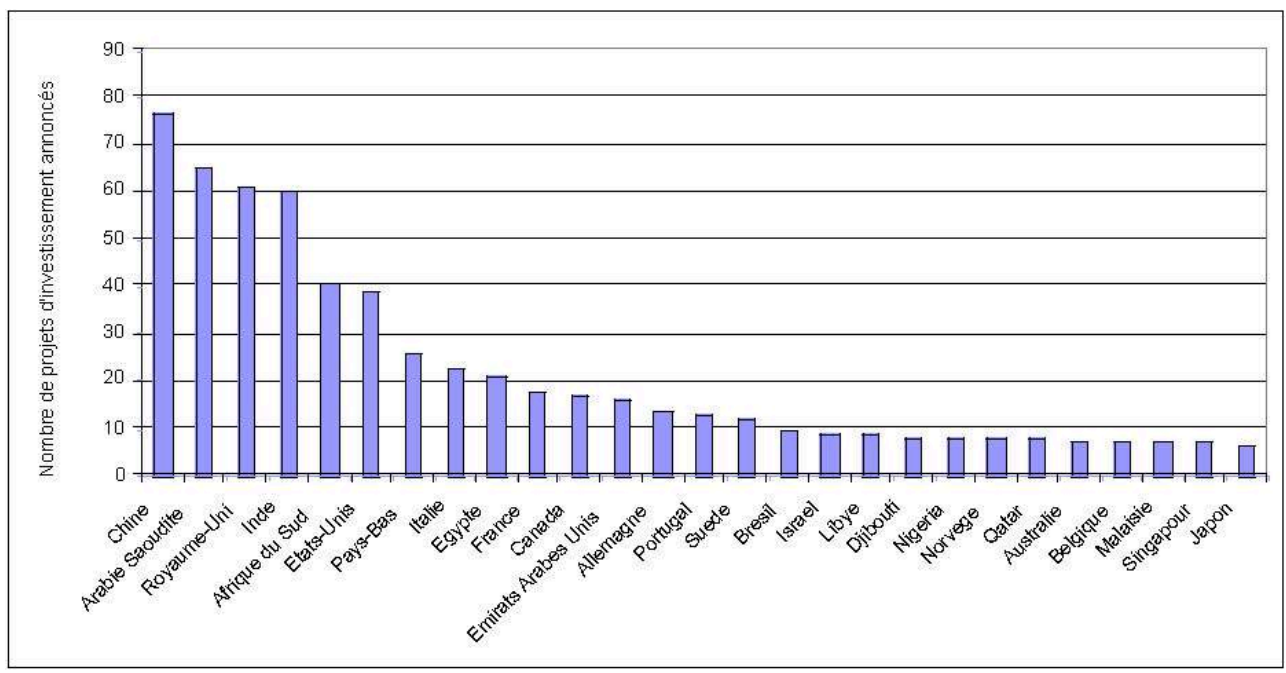

Les pays investisseurs dans l'agriculture et le foncier en Afrique

Source : Anseeuw \& Taylor, 2011.

On remarque trois catégories d'acteurs impliqués dans ces dynamiques d'investissement foncier et agricole.

Tout d'abord, certains États inquiets de leurs sécurité alimentaire ont développé des stratégies d'acquisition foncière ou d'investissements agricoles massifs à l'étranger. Il s'agit ainsi d'assurer leur approvisionnement en denrées alimentaires en délocalisant leur production nationale afin de ne plus dépendre des marchés internationaux. Jusqu'à aujourd'hui, ces principaux investisseurs étaient situés en Asie du Sud-Est, notamment en Chine, en Corée et en Inde, mais aussi au Moyen-Orient. Dans ce cas de figure, le gouvernement est directement impliqué dans ces opérations à travers des fonds d'investissement souverains (pour les pays du Golfe, prenons l'exemple de l'initiative «King Abdullah for Saudi Agricultural Investment Abroad» de l'Arabie saoudite) ou par l'intermédiaire d'entreprises (para-) publiques (comme la Chine dans le cadre de sa politique « going global»).

La deuxième catégorie d'investisseurs est celle des multinationales agroalimentaires occidentales. Ces entreprises cherchent à élargir leur contrôle sur tous les segments de la chaîne de production, notamment vers la production primaire. En effet, selon Cotula et Vermeulen ${ }^{8}$, on assiste actuellement à un retournement du rapport risque/bénéfice au sein de la chaîne de production. Si la production primaire concentrait jusqu'à présent l'essentiel des risques tandis que les bénéfices revenaient aux acteurs en amont mais surtout en aval, la hausse des prix agricoles tend désormais à inverser cette tendance. Ainsi, en intégrant directement la production primaire, ces entreprises agroalimentaires cherchent à élargir leurs parts de marché et à renforcer leur positionnement au sein des différentes filières.

Enfin, la troisième catégorie d'acteur, celle qui nous intéresse plus spécialement dans cet article, regroupe les investisseurs-spéculateurs. La crise de 2008 conjuguée aux projections démographiques et économiques (agrocarburant, hausse de la population mondiale...) a bousculé les idées reçues sur le secteur agricole. Aujourd'hui, en vertu des anticipations dominantes, le secteur agricole est perçu comme un investissement sur l'avenir. Ainsi, des acteurs totalement étrangers à l'agriculture ont commencé à s'y intéresser et à y investir des capitaux afin de profiter de la hausse annoncée des prix 
des denrées agricoles et de la terre à vocation agricole. Des banques d'investissement, des fonds de pension et des hedge funds investissent désormais dans le secteur agricole, tout au long des chaînes de production, réalisant ainsi une «spéculation d'orientation malthusienne ».

12 Cette prise de contrôle croissante sur le cycle productif agricole, et notamment la production primaire, se fait à travers une intégration verticale renforcée. Les segments en amont (y compris le financement) et en aval (jusqu'à la distribution) subissent un processus de concentration de plus en plus poussé qui se fait au bénéfice de quelques macro-acteurs. Par le biais de partenariats, de contractualisation, etc., ces acteurs dominants étendent leur contrôle sur la production primaire et sur le cycle productif dans sa totalité. Cette intégration s'effectue généralement par l'intermédiaire de la chaîne de valeur financière (value chain finance), vecteur par lequel s'effectue cet apport de capitaux.

«La chaîne de valeur financière structure les financements qui sont proposés tout au long de la chaîne de valeur. Les services financiers sont souvent combinés avec des activités de commercialisation et éventuellement d'assistance technique. ${ }^{9}$

14 Cette approche n'est pas nouvelle, et plusieurs filières d'exportation (café, coton...) sont déjà structurées autour de ce modèle. Cependant, ces dernières années, cette stratégie financière a connu une diffusion plus large, aussi bien au niveau géographique qu'au niveau des filières concernées (ex.: les céréales). Cette approche vise également à limiter les risques inhérents à l'activité agricole. Grâce à une circulation des flux de capitaux et d'informations, les coûts de transaction sont réduits et les risques limités.

Les investisseurs financiers (investisseurs-spéculateurs) utilisent cette chaîne de valeur pour prendre position au sein des filières de production. Détaillons maintenant les modalités de cette prise de contrôle en fonction des caractéristiques des investisseurs.

Sans prétendre être exhaustif, nous présentons ici trois modèles: l'intégration bancaire, l'engagement des compagnies d'ingénierie et les fonds d'investissement ${ }^{10}$.

17 Les banques sont des partenaires traditionnels de l'agriculture et de l'agro-industrie, qu'elles financent sous une forme " classique » caractérisée par un prêt accordé avec un bien en garantie, généralement la terre. Dans un contexte de perspectives de rendements financiers accrus, les banques tendent à présent à renforcer leur contrôle et leur participation le long des chaînes de production agricole. Cette intégration bancaire se fait essentiellement par la contractualisation des différentes parties, et notamment les producteurs (Figure 2).

18 Concernant leurs relations avec les producteurs, on voit apparaittre une nouvelle stratégie de gestion du risque. La production va désormais couvrir le prêt concédé au lieu de la terre. Cette évolution doit être mise en parallèle avec l'augmentation du coût des intrants par rapport à la valeur la terre. Souvent, cette dernière n'est plus une garantie suffisante pour couvrir les dépenses des agriculteurs. Ainsi, la banque fournit les liquidités nécessaires aux producteurs pour l'achat des intrants (graines, fertilisants, etc.) en échange des droits sur la récolte future. Ce contrat est négocié entre les deux parties au début du cycle productif, autrement dit, avant même que les graines ne soient mises en terre, l'agriculteur a perdu la possession de sa production.

19 Le contrat stipule le type, le volume et la qualité de la production, définis en fonction des caractéristiques de l'exploitation (taille, qualité du sol...), et le prix d'achat par la banque. Ce prix d'achat est calculé en fonction des projections d'évolution du marché. 
Le producteur dispose donc d'un revenu fixe défini à l'avance, à savoir le montant de la production moins le prêt concédé et les intérêts. En cas de surplus ou de manque par rapport au volume et à la qualité fixés, l'agriculteur sera crédité ou débité du montant correspondant. Le risque de production est donc transformé en risque de performance, lequel est intégralement supporté par l'agriculteur.

La banque prend en charge une assurance multi-périls qui couvre le producteur contre tous les risques naturels inhérents à l'activité agricole (inondation, incendie...) et ce, dès le début du cycle. Parce qu'elle contractualise avec plusieurs exploitants dispersés géographiquement, la banque limite les risques de production et bénéficie d'importantes économies d'échelle auprès des compagnies d'assurance. Elle limite également les risques de fluctuation des prix en se chargeant de la gestion et de la couverture des prix grâce au marché à terme des produits agricoles (le marché à terme SAFEX à Johannesbourg).

21 Au cours du cycle productif, la banque et la compagnie d'assurance vont effectuer un suivi régulier de la production qui sera assuré par des ingénieurs agronomes, employés par les banques, mais aussi par satellite. À la fin de la récolte, l'agriculteur livre sa production à un silo certifié par le marché à terme, lequel transmet à la banque un reçu garantissant la possession d'un volume et d'une qualité précis. La banque se charge alors d'écouler la production physique principalement par le marché financier.

Figure 2

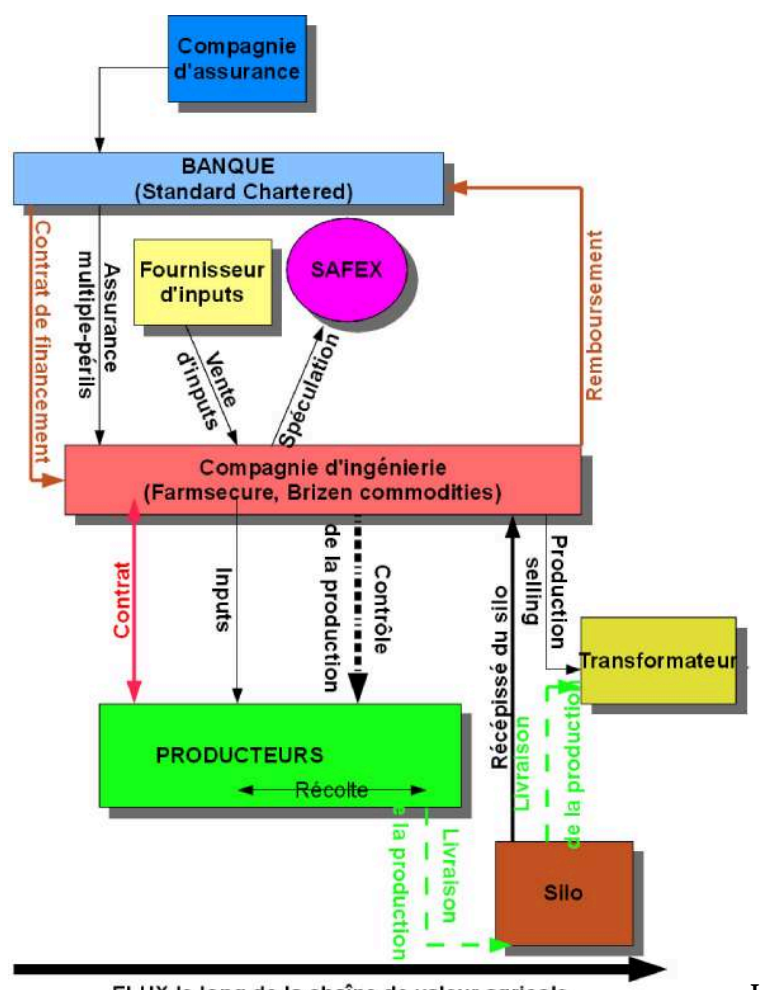

FLUX le long de la chaîne de valeur agricole

L'intégration bancaire dans les

chaînes de valeur agricole

Source : Ducastel, 2010.

On peut estimer que 30 à $40 \%$ de la production annuelle de céréales en Afrique du Sud est réalisée dans le cadre de ces modèles. En effet, les trois principales banques commerciales (ABSA, Standard Chartered et RMB) déclarent détenir chacune environ 
$13 \%$ de la production. Elles possèdent rarement plus pour limiter encore le risque. Ces banques diversifient à présent leurs investissements agricoles vers l'horticulture, l'élevage et d'autres filières de production.

L'intégration de la filière peut également se faire par des intermédiaires des sociétés d'ingénierie agricole, dont la vocation est de centraliser tous les services destinés aux agriculteurs (fourniture d'intrants, assistance technique, vente de la production) au sein d'une même entité. Elles proposent donc aux producteurs un arrangement contractuel qui se veut une solution intégrale.

Dans la grande majorité des cas, il n'y a pas de transfert ou d'acquisition de foncier. Le producteur loue sa terre à la compagnie de gestion, souvent pour une saison. Des objectifs de production sont fixés dans le contrat, ainsi que le cahier des charges et la rémunération correspondante pour le producteur. Le producteur n'a aucun droit de regard sur la prise de décision quant à l'utilisation de son exploitation. En échange, la société fournit directement les intrants aux exploitants et garantit le prix de vente via l'acquisition de positions sur le marché à terme. Au cours du cycle de production, cette compagnie assure un suivi sur les exploitations. Des ingénieurs sont envoyés sur le terrain, les opérations sont supervisées grâce à un système satellite de pointe et les comptes du producteur sont surveillés. Après la récolte, la compagnie prend en charge la commercialisation de la production dont elle détient les droits. Une fois la production écoulée, la compagnie de gestion rembourse le prêt octroyé par la banque avec les intérêts (Figure 3).

Dans ce modèle, les relations financières ont également changé voire ont disparu pour le producteur au profit de celles qui lient la banque et l'entreprise associée. Elles sont alors définies par un contrat qui stipule que la compagnie intermédiaire est à la fois le garant du prêt saisonnier et le superviseur de la production. La banque lui fournit donc les liquidités et les assurances multi-périls nécessaires.

La plus-value d'un tel système par rapport au précédent réside dans la spécialisation agricole et la proximité de l'entreprise avec les acteurs de la filière. Cette compagnie réalise ses bénéfices grâce à l'apport de technologies de pointe dans les exploitations, en effectuant des économies d'échelle pour l'achat des intrants, les assurances, etc., et grâce à une gestion poussée de l'ensemble des risques que la banque lui a délégués : le marché financier/agricole à terme pour garantir un prix de vente, les assurances pour les risques naturels. En outre, en sélectionnant les producteurs en fonction de leur expérience et des caractéristiques de leur exploitation, puis en assurant un contrôle sur le terrain, la compagnie réduit les risques liés au volume et à la qualité de la production. Finalement, ces sociétés tendent à mener une double stratégie de diversification. D'une part, comme dans le modèle précédent, elles passent des contrats avec des fermes situées dans des zones géographiques différentes; d'autre part, elles développent leurs activités dans plusieurs filières agricoles. Si les céréales font partie de leurs cibles prioritaires, elles n'hésitent pas à s'engager dans la production de produits frais, de biocarburants ou encore de viandes.

Il est à ce jour difficile d'évaluer l'étendue de ce modèle. La société d'ingénierie agricole la mieux établie est Farmsecure - entreprise sans expérience préalable dans le secteur agricole, créée, en 2004, avec l'objectif de reprise de petites et moyennes entreprises -, qui dispose de 700 exploitations en Afrique du sud, représentant près de $8 \%$ de la production annuelle de céréales. D'autres sociétés semblent se structurer rapidement. 
Figure 3

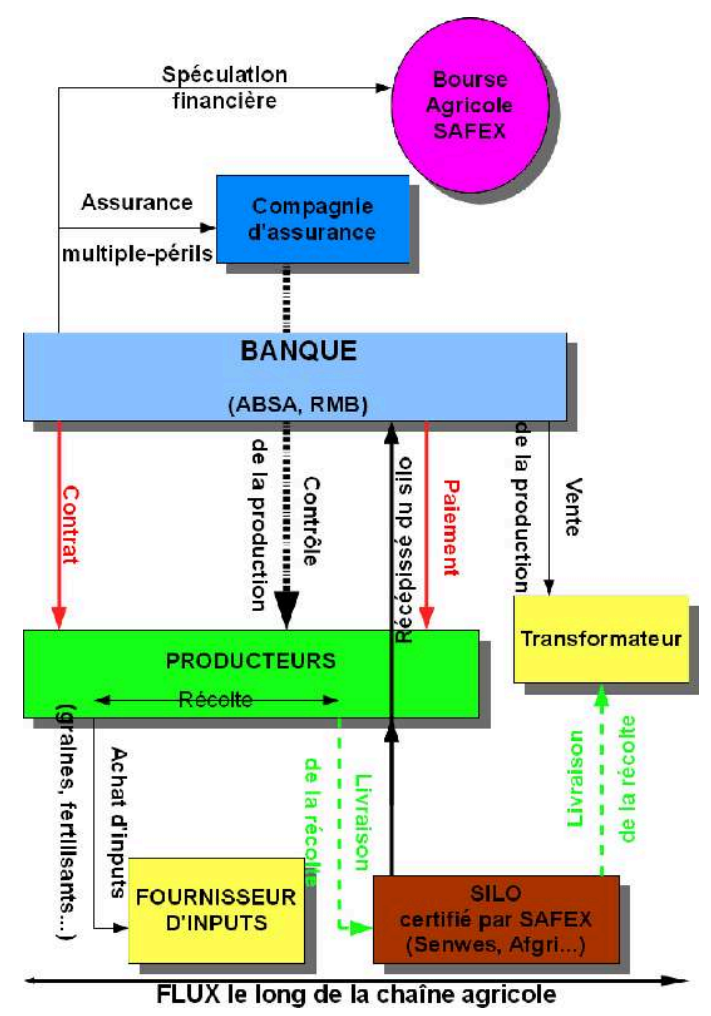

Le modèle des sociétés d'ingénierie agricole

Source : Ducastel, 2010.

Depuis 2008, le secteur agricole sud-africain est également marqué par la prolifération de fonds d'investissement spécialement dédiés à l'agriculture. Le profil de ces fonds et des investisseurs qui y contribuent est divers: banques commerciales, investisseurs institutionnels (fonds de pension), acteurs publics (agences de développement), etc.; mais, pour la plupart, il s'agit d'acteurs extérieurs au secteur. La gestion de ces fonds est généralement confiée à des sociétés d'ingénierie agricole qui disposent d'une expérience et d'un réseau local.

En fonction des attentes des investisseurs (taux de rentabilité, durée de vie du fonds, etc.) et de leurs anticipations sur l'avenir, ces fonds vont adopter différentes stratégies. Tous ne vont pas cibler les mêmes actifs, ni adopter la même gestion de ces actifs. Ainsi, certains vont se spécialiser dans l'acquisition foncière comme, par exemple, Emvest et African/South African Agricultural Fund. Dans ce cas de figure, ces structures cherchent à acquérir, sous la forme d'achat ou de prêt sur le long terme, des terres arables à potentiel agricole. Dans cette catégorie de fonds d'investissement dédiés au foncier, on peut distinguer ceux qui prennent directement en charge la production sur ces exploitations par opposition à ceux qui vont l'externaliser. La première catégorie mise sur une hausse de la productivité grâce à l'utilisation de technologies de pointe notamment et sur la hausse des prix des denrées agricoles. Le deuxième groupe va louer la terre à des agriculteurs en charge de sa mise en valeur et parier davantage sur la hausse de son prix, et du loyer qu'ils perçoivent. Deux types de spéculation soutiennent cette dynamique, l'une sur le foncier et l'autre sur les produits agricoles.

Certains acteurs souhaitant investir dans le secteur agricole considèrent cette stratégie d'acquisition directe du foncier, avec ou sans prise en charge de la production, comme 
trop risquée. Dans ce cas, le fonds d'équité constitue une alternative. Ce dernier a pour objectif l'acquisition d'actions (equities) d'une entreprise agricole ou agro-industrielle. Le risque est limité puisque les capitaux ne sont pas placés dans des actifs aussi rigides et est politiquement et socialement moins sensible que le risque lié à la terre. L'entreprise recevant ces fonds jouit pour sa part d'un apport de capitaux conséquent qui lui permet de développer ses activités. Si ce mode de financement est moins coûteux que le prêt pour l'entreprise, elle cède en échange une partie de son autonomie décisionnelle à l'investisseur.

31 Tout comme ceux dédiés à l'acquisition de terres, ces fonds d'investissement en actions mènent différentes stratégies guidées par les prophéties auxquelles elles adhèrent et, surtout, par les attentes de leurs investisseurs. Par exemple, les «fonds à durée déterminée " ont une durée de vie allant de dix à quinze ans, voire plus courte, et garantissent à leurs clients des rendements élevés à court terme. Ils vont alors privilégier les entreprises et les domaines d'activité où les marges sont potentiellement les plus importantes et les plus rapides pour un investissement minime. En revanche, les fonds qui n'ont pas de «date de péremption » adoptent des stratégies basées sur le plus long terme avec des retours garantis et réguliers.

On constate aussi que les fonds d'investissement en actions privilégient les prises de position au sein d'entreprises déjà rentables et concurrentielles. Ces dernières vont bénéficier de l'apport de capitaux pour se renforcer. Ainsi, on peut penser que cette intrusion de plus en plus massive de fonds d'investissement vient renforcer la position des acteurs dominants dans le secteur agricole, au détriment des autres. Selon que la position acquise est majoritaire ou minoritaire, le fonds d'investissement n'aura pas le même droit de regard et de gestion des activités de la compagnie. Zeder, par exemple, pour des raisons de risques et ne voulant pas s'impliquer dans la stratégie et la gestion de la production, ne vise que des positions minoritaires (entre 20 et $34 \%$ des parts). À l'inverse, Agri-Vie tend à contrôler l'ensemble des activités de ses filiales en leur imposant son propre modèle de gestion (Tableau 1).

Tableau 1

\begin{tabular}{|c|c|c|c|c|c|}
\hline $\begin{array}{c}\text { Fonds } \\
\text { d'investissement } \\
\text { (date de } \\
\text { création) }\end{array}$ & $\begin{array}{l}\text { Détenteur du } \\
\text { fonds }\end{array}$ & $\begin{array}{c}\text { Origine des } \\
\text { capitaux }\end{array}$ & $\begin{array}{c}\text { Montant de } \\
\text { la } \\
\text { capitalisation }\end{array}$ & $\begin{array}{c}\text { Stratégie } \\
\text { d'investissement }\end{array}$ & $\begin{array}{c}\text { Zone } \\
\text { d'activité }\end{array}$ \\
\hline $\begin{array}{l}\text { Emvest } \\
(2008)\end{array}$ & $\begin{array}{c}\text { Emergent Asset } \\
\text { Management } \\
\text { (fonds } \\
\text { d'investissement } \\
\text { britannique } \\
\text { spécialisé dans } \\
\text { les marchés } \\
\text { émergents) \& } \\
\text { Russel Stone } \\
\text { Group (société } \\
\text { agro-industrielle } \\
\text { sud africaine) }\end{array}$ & & & \begin{tabular}{|lr} 
Acquisition & \\
foncière & avec \\
prise en charge \\
directer de & la \\
production, de la \\
transformation & et \\
de & la \\
commercialisation \\
Diversification \\
des filières
\end{tabular} & Afrique \\
\hline
\end{tabular}




\begin{tabular}{|c|c|c|c|c|c|}
\hline $\begin{array}{l}\text { South African } \\
\text { agricultural fund } \\
\& \text { African } \\
\text { agricultral fund } \\
\text { (2010) }\end{array}$ & $\begin{array}{l}\text { Old mutual } \\
\text { (institution } \\
\text { financière sud- } \\
\text { africaine) }\end{array}$ & $\begin{array}{l}\text { Compagnies } \\
\text { d'assurance } \\
\text { vie et fonds de } \\
\text { pension } \\
\text { européens et } \\
\text { sud-africains }\end{array}$ & $\begin{array}{l}3 \text { milliards de } \\
\text { rands chacun } \\
\text { (environ } \\
300 \text { millions } \\
\text { d'euros) }\end{array}$ & $\begin{array}{l}\text { Acquisition } \\
\text { foncière à des } \\
\text { fins spéculatives } \\
\text { (pas de contrôle } \\
\text { direct sur la } \\
\text { production }\end{array}$ & $\begin{array}{l}\text { Afrique } \\
\text { australe }\end{array}$ \\
\hline $\begin{array}{l}\text { Zeder } \\
(2006)\end{array}$ & $\begin{array}{l}\text { PSG (groupe } \\
\text { sud-africain } \\
\text { dédié aux } \\
\text { services } \\
\text { financiers) }\end{array}$ & & & $\begin{array}{l}\text {-Position } \\
\text { minoritaire (entre } \\
20 \text { et } 34 \% \text { ) } \\
\text {-Pas d'implication } \\
\text { directe dans la } \\
\text { production mais } \\
\text { droit de regard au } \\
\text { niveau } \\
\text { managérial } \\
\text {-Activité en } \\
\text { amont et en aval }\end{array}$ & $\begin{array}{l}\text { Afrique du } \\
\text { sud }\end{array}$ \\
\hline $\begin{array}{l}\text { Agri-Vie } \\
(2008)\end{array}$ & $\begin{array}{c}\text { Sanlam (société } \\
\text { d'assurance sud- } \\
\text { africaine) }\end{array}$ & $\begin{array}{l}\text { Fonds de } \\
\text { pension, } \\
\text { fondations } \\
\text { privées } \\
\text { (Kellogs), } \\
\text { institutions } \\
\text { publiques } \\
\text { (Industrial } \\
\text { Development } \\
\text { Corporation) }\end{array}$ & $\begin{array}{l}700 \text { millions de } \\
\text { rands } \\
\text { (70 millions } \\
\text { d'euros) }\end{array}$ & $\begin{array}{ll}\text {-Acquisition de } & \\
\text { position } & \\
\text { majoritaire au } & \\
\text { sein } & \\
\text { compagnies } & \\
\text { agricoles et agro- } \\
\text { industrielles } \\
\text { (céréales, } \\
\text { élevage, } \\
\text { horticulture...) } \\
\text {-Contrôle direct } \\
\text { de la production } \\
\text { Priorité à la } \\
\text { production } \\
\text { primaire }\end{array}$ & $\begin{array}{l}\text { Continent } \\
\text { africain }\end{array}$ \\
\hline $\begin{array}{l}\text { African Agricultural } \\
\text { fund } \\
(2009)\end{array}$ & AFD (?) & $\begin{array}{c}\text { AFD, BAD, } \\
\text { AGRA, IFAD, } \\
\text { Banque ouest- } \\
\text { africaine de } \\
\text { développement }\end{array}$ & $\begin{array}{c}150 \text { millions de } \\
\text { dollars }\end{array}$ & $\begin{array}{l}\text {-Intégralité de la } \\
\text { chaine de } \\
\text { production } \\
\text { agricole } \\
\text { (production } \\
\text { primaire, } \\
\text { transformation, } \\
\text { infrastructures...) } \\
\text {-Vers l'agriculture } \\
\text { commerciale } \\
\text { (80\% } \\
\text { capitaux) des } \\
\text { l'agriculture et } \\
\text { familiale (20\%) }\end{array}$ & $\begin{array}{l}\text { Continent } \\
\text { africain }\end{array}$ \\
\hline $\begin{array}{l}\text { TransFarm Africa } \\
\qquad(2011)\end{array}$ & $\begin{array}{l}\text { NEPAD business } \\
\text { fondation }\end{array}$ & $\begin{array}{c}\text { Fondations } \\
\text { privées } \\
\text { (Hewlett)... }\end{array}$ & $\begin{array}{l}20 \text { millions de } \\
\text { dollars }\end{array}$ & $\begin{array}{l}\text { Stratégie pas } \\
\text { encore } \\
\text { développée }\end{array}$ & $\begin{array}{l}\text { Continent } \\
\text { africain }\end{array}$ \\
\hline
\end{tabular}




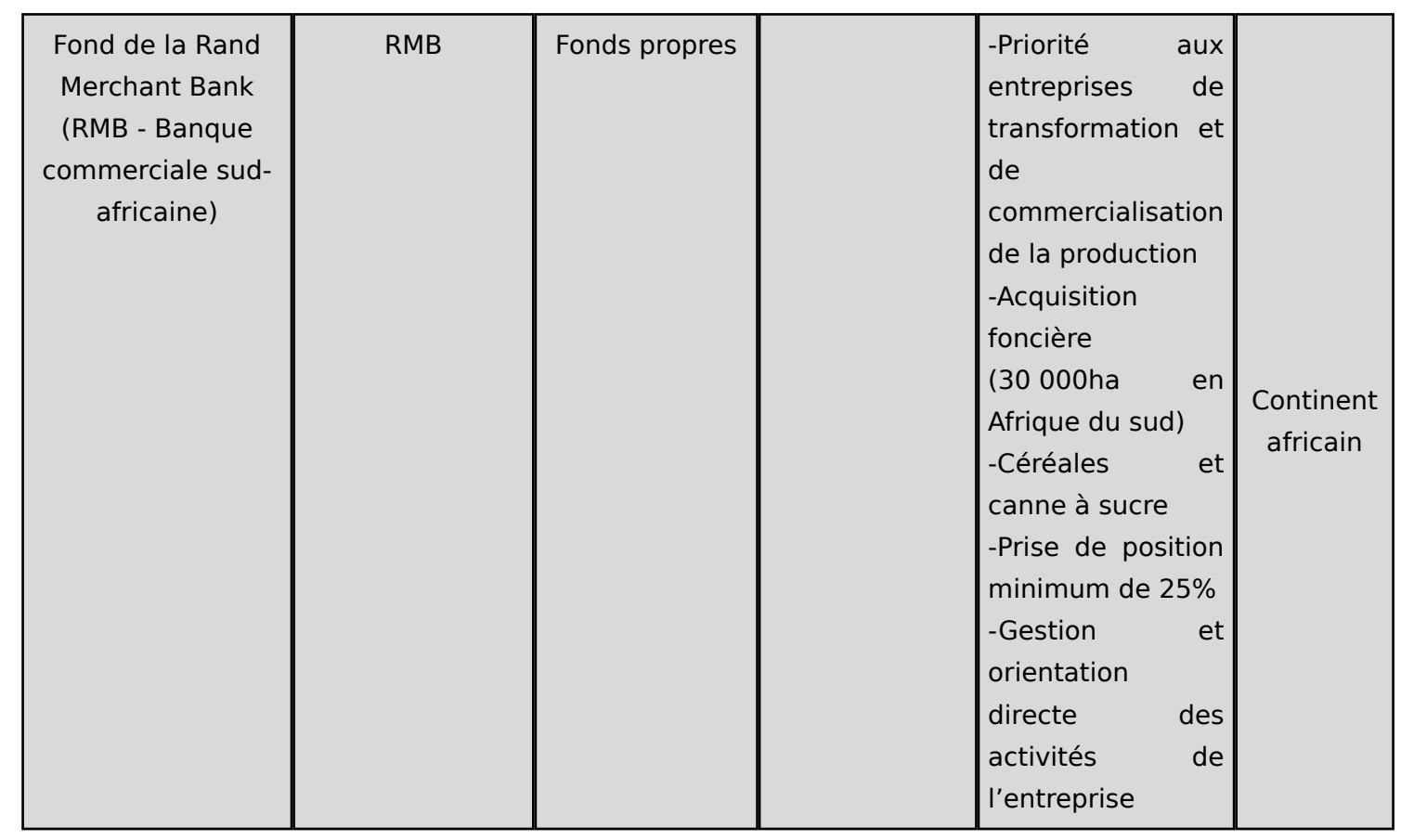

Quelques exemples d fonds d'investissements spécialisés dans le domaine agricole en Afrique du Sud Ducastel, 2010

33 Au sein des investisseurs, il est fréquent de retrouver des institutions publiques telles que des agences de développement nationales ou internationales et des fondations privées, dédiées au développement. Souvent, au sein d'une même entité, cohabitent ainsi une orientation commerciale et une volonté de développement. Cette mixité des investisseurs vient illustrer la confusion croissante entre promotion du développement et promotion de l'investissement privé.

34 L'étendue de ces investissements est inconnue, d'autant plus que plusieurs fonds ont été créés récemment et n'ont pas encore de stratégie de mise en œuvre (c'est le cas de TransFarm Africa).

35 Cet état des lieux de l'agriculture sud-africaine à partir des modèles de financement en vigueur montre plusieurs tendances et questionnements dans une perspective africaine.

Le secteur agricole mondial subit actuellement une profonde restructuration. L'exception agricole qui avait été remise en cause avec l'entrée du secteur dans les négociations du GATT est bel et bien enterrée. De nouveaux acteurs, porteurs de références et d'expériences extérieures, ont fait leur apparition. De leurs interactions avec les agents « traditionnels » naissent de nouveaux modes d'action, d'investissement et de production. Ainsi, un nouveau paradigme du développement agricole tend aujourd'hui à s'imposer ${ }^{11}$, dont les répercussions se manifestent à la fois sur la scène internationale et dans les espaces nationaux.

37 Tout d'abord, cette étude montre que de nouveaux acteurs apparaissent sur la scène agricole africaine. Qu'ils soient issus des domaines industriels ou financiers, qu'ils soient des entrepreneurs, des investisseurs ou même de purs spéculateurs, les pourvoyeurs de capitaux semblent de plus en plus exogènes au monde agricole. Outre des financements, ces acteurs apportent une logique, des moyens d'action et de 
régulation, issus d'autres sphères d'activité. On assiste en quelque sorte à une " vassalisation du secteur » qui se traduit par une redéfinition des frontières de l'espace agricole. Le rôle croissant des banques et des fonds d'investissement, par exemple, semble conduire à une « financiarisation » du secteur.

Le secteur agricole sud-africain connaît aussi actuellement un processus d'industrialisation, ou plutôt de «corporisation $»^{12}$. Ce n'est pas tant la mécanisation qui constitue la spécificité d'une telle dynamique mais plutôt la transformation des structures de production. De plus en plus, les chaînes de valeur agricole sont contrôlées par quelques acteurs dominants. Cette prise de contrôle des différents segments le long de cette chaîne se fait soit par acquisition directe, soit par la contractualisation des acteurs. Si, en Afrique du Sud, les banques et certaines des ex-coopératives jouent ce rôle d'acteurs dominants, cette tendance se retrouve aussi ailleurs sous d'autres formes (ex. : l'Uruguay $\left.{ }^{13}\right)$. L'organisation de la production agricole se dirige vers une structure fortement intégrée, proche des chaînes tayloristes industrielles, dans lesquelles les segments les plus risqués sont externalisés vers des intermédiaires qui en supportent les coûts et les risques.

La financiarisation et la corporisation mènent également à une ouverture globale du secteur. Deux tendances peuvent être mises en avant.

D'une part, le capital agricole investi est autant étranger que domestique. Ceci est surtout le cas pour le secteur bancaire qui contrôle une partie significative de certaines filières agricoles (en particulier les céréales et certains oléagineux comme le soja). La banque ABSA est détenue à 56,4\% par le groupe financier anglais Barclays ; Standard Bank (aussi connue pour ses activités agricoles en dehors de l'Afrique du Sud) appartient pour $20 \%$ à la Banque industrielle et commerciale de Chine, ICBC. Mais cette ouverture financière internationale concerne de même les fonds d'investissement et les entreprises d'ingénierie agricole (voir tableau 1). C'est le cas du fonds Emvest dont les capitaux proviennent de la société de gestion d'actifs londonienne Emergent Asset. Parmi les principaux clients d'Emergent, on trouve les universités américaines Harvard ou encore Vanderbilt, qui auraient investi près de 500 millions de dollars dans Emvest ${ }^{14}$.

D'autre part, les activités et le capital sud-africain (ou opérant de l'Afrique du Sud) investissent d'autres marchés surtout africains. Plusieurs indices laissent entrevoir que ces modèles de production et d'investissement se propagent sur le reste du continent. Les sociétés sud-africaines agricoles et agro-industrielles se lancent à la conquête de nouveaux marchés. Ainsi, Farmsecure est déjà présente dans dix-huit pays africains et ambitionne de poursuivre son expansion, notamment vers l'Afrique de l'Ouest; les banques sud-africaines sont, elles aussi, de plus en plus présentes sur tout le continent (Standard Bank est présente dans quatorze pays et s'investit particulièrement dans le domaine agricole; RMB acquiert des parts de banques africaines afin de réorienter leurs activités); et depuis quelques mois, plusieurs projets de cultures à grande échelle ont été annoncés par des sociétés sud-africaines dans des pays africains (ex. : AgriSA en République démocratique du Congo $)^{15}$. De plus, d'autres initiatives internationales et africaines développent leurs activités sur des modèles similaires : banque néerlandaise RaboBank déjà engagée dans le secteur agricole d'une dizaine de pays ; prospection de banques chinoises auprès des entreprises sud-africaines; fonds d'investissement kenyan présent dans plusieurs pays de l'Afrique de l'Est. Si, le plus souvent, les conditions d'investissement ne sont pas aussi propices qu'en Afrique du Sud, des mesures sont prises afin de s'adapter aux conditions locales: on assiste à des 
investissements dans la production d'intrants, afin de développer un modèle de financement sans transfert de liquidités, et ainsi limiter les risques dans des environnements moins propices que l'Afrique du Sud, ou encore à l'implantation de prototypes de silos facilement transportables et adaptés à différents climats pour les pays où de telles infrastructures sont peu présentes.

La forte volatilité des prix agricoles renforcée par la suppression des mécanismes de stabilisation, dans un contexte de régulation du secteur par le marché, pose la question du choix des agents économiques et des mécanismes de régulation agricole. Au cours des dernières années, la spéculation agricole a connu un "boom " sans précédent. Si auparavant la spéculation était un phénomène interne et de courte durée, elle adopte désormais des stratégies sur le long terme, menées par des acteurs étrangers au secteur. Ainsi, dans le cadre des échanges sur les marchés à terme agricoles (la SAFEX en Afrique du Sud), de moins en moins de contrats débouchent sur une livraison effective.

D'un côté, la spéculation peut renforcer des stratégies d'accroissement des profits, au détriment de la sécurité alimentaire du pays où a lieu la production. D'un autre côté, les pouvoirs économiques étrangers contrôlant une part croissante de la production mettent également en cause la souveraineté alimentaire de ces pays, dans un contexte de libéralisation poussée. La sécurité et la souveraineté alimentaires nationales sont donc en jeu. En effet, comme le note le rapporteur spécial sur le droit à l'alimentation, une part significative de la volatilité et de la hausse des prix s'explique par l'arrivée de la spéculation, et un rôle primordial est joué ici par l'entrée sur les marchés de puissants investisseurs institutionnels (fonds spéculatifs, fonds de pension, banques d'investissement) ${ }^{16}$, souvent étrangers et généralement peu intéressés par des objectifs de stabilisation, de sécurité et de souveraineté alimentaire.

Si cette volatilité des cours des produits agricoles et les stratégies de spéculation posent problème pour la mise en place de programmes de développement, elles soulèvent aussi des questions sur la régulation des secteurs agricole et financier et sur les cadres réglementaires dans un grand nombre de domaines allant jusqu'au fonctionnement des marchés à terme et au commerce extérieur. Cela suscite également des réflexions autour des politiques nationales, du développement des stratégies sectorielles et financières, et de l'intégration régionale.

En effet, les résultats présentés dans cet article montrent que deux groupes d'acteurs semblent particulièrement bénéficier de la restructuration agricole. Tout d'abord, les banques commerciales et les compagnies d'assurances qui, en contrôlant directement une partie croissante de la production primaire et en imposant leurs critères de sélection aux exploitants, se posent en régulateurs du secteur. En intégrant toute la filière et en centralisant les flux d'informations, ils peuvent anticiper l'évolution de ces marchés et notamment les prix, jouer le rôle d'arbitre ${ }^{17}$ et faire évoluer les prix en leur faveur en spéculant à la hausse ou à la baisse selon les anticipations. Ensuite, les autres grands bénéficiaires de l'évolution des structures de production sont les intermédiaires agricoles : les institutions financières souhaitant s'investir dans le domaine agricole ont de plus en plus recours aux services de sociétés d'ingénierie agricole. À la fois gestionnaires des opérations de terrain et des opérations financières, ces sociétés captent une part croissante des marges générées par l'activité de production agricole.

Ce double processus, à la fois de financiarisation et de "corporisation» du secteur agricole, conduit à un nouveau régime qui se caractérise par la domination de quelques 
grands groupes agroalimentaires internationaux ${ }^{18}$ et l'exclusion de la majorité des agriculteurs africains confrontés à des modèles à productivité significativement plus élevée ${ }^{19}$.Ces évolutions renforcent la dualisation et la concentration de la structure agricole sud-africaine. La majorité de la population rurale est exclue de ces dynamiques dont les coûts d'entrée se révèlent trop élevés. Les paysans qui parviennent à s'insérer dans ces schémas sont, quant à eux, réduits à l'état de prestataires de services agricoles. En effet, ils n'ont pas les mêmes ressources financières, sociales et culturelles pour négocier sur un pied d'égalité avec les firmes ${ }^{20}$. Ainsi, c'est toute l'organisation socioéconomique du monde agricole qui subit le choc de ces transformations.

Ces évolutions suscitent l'inquiétude, d'autant qu'en l'absence de modèles alternatifs, la conception du développement agricole focalisée sur des macro-acteurs devient le paradigme de référence, en Afrique du Sud mais également sur le reste du continent. Elle est en effet adoptée par les agences publiques de développement (NEPAD, BAD, etc.) et exportée par ces macro-acteurs dans le cadre de leur expansion économique sur le continent. On ne peut alors que déplorer le manque de réflexions et de débats politiques autour des trajectoires de développement, qu'elles soient agricoles ou non, pour ces pays à la recherche d'alternatives.

\section{NOTES}

1. OCDE, « Afrique : Priorité à l'agriculture », Paris, OCDE, 2010, www.oecd.org/agriculture.

2. Cette hausse des dépenses agricoles se fait dans le cadre de l'accord de Maputo. Dans cet accord, signé par tous les États africains, en 2004, et lié au Programme détaillé pour le développement de l'agriculture africaine (PDDAA) du Nouveau partenariat pour le développement africain (NEPAD), les États africains s'engagent à consacrer $10 \%$ de leur budget national à l'agriculture. Cependant, jusqu'en 2008, quatre pays seulement s'y étaient effectivement engagés.

3. OXFAM, «D'un G8 à l'autre : suivi des engagements de l'Aquila sur la sécurité alimentaire », note d'information, Paris, OXFAM-France, 24 juin 2010.

4. W. Anseeuw, L. Alden-Wily, L. Cotula et M. Taylor, «Pressions commerciales sur la terre », rapport de recherche, Rome, International Land Coalition (à paraître).

5. Voir Business news, $1^{\text {er }}$ septembre 2010. Dans cet article, Neil Crowther, chef de la direction de l'entreprise d'investissement Chayton Capital, déclara également : «les investissements d'équité privés dans le secteur agricole africain ont généré parmi les retours sur investissement les plus élevés l'année dernière ».

6. UNCTAD, « Rapport sur l'investissement dans le monde : sociétés transnationales, production agricole et développement en 2009 », rapport - vue d'ensemble, New York/Genève, UNCTAD, 2009, $69 \mathrm{p}$.

7. L. Cotula, S. Vermeulen, R. Leonard and J. Keeley, Land Grab or Development Opportunity? Agricultural Investment and International Land Deals in Africa, research report, London/Rome, IIED/FAO/IFAD, 2009, 130 p.; World Bank, «Rising Global Interest in Farmland. Can it yield sustainable and equitable benefits?", research report, Washington, WB, 2010, 194 p.; W. Anseeuw, L. Alden-Wily, L. Cotula et M. Taylor, op. cit. ; etc. 
8. L. Cotula and S. Vermeulen, «Deal or no deal: the outlook for agricultural land investment in Africa », International Affairs, vol. 85, nº, 2009 : 1233-1247.

9. J.-C. Devèze, Défis agricoles africains, Paris, Karthala/AFD, 2008.

10. Pour des descriptions plus détaillées de ces modèles, voir A. Ducastel, «La restructuration du secteur agricole en Afrique du Sud », mémoire de master 2, Paris, université Paris-I Sorbonne, CIAHPD, 2010, $91 \mathrm{p}$.

11. A. De Janvry, "Agriculture for Development: new paradigm and option for success", Agricultural Economics, vol. 41, Issue supplement s1, 2009 : 17-36.

12. Terme anglo-saxon qui désigne le processus par lequel une entité tend à acquérir la forme organisationnelle ainsi que les modes d'action propres à une entreprise.

13. FAO, «Análisis de cadenas basadas en la producción de granos de secano para la definición de lineamientos de políticas específicas en Uruguay ", research report, Rome, FAO, 2009.

14. Oakland Institute, "Understanding Land Investment Deals in Africa ", research report, Oakland, Oakland Institute, 2011; " US Universities in Africa 'land grab' », Guardian UK, 6 août 2011.

15. R. Hall, "The next great trek? South African commercial farmers move North. Cape Town ", PLAAS, working paper, $\mathrm{n}^{\circ} 19,2011,17 \mathrm{p}$.

16. «Spéculation agricole et flambée des prix alimentaires. Réguler pour réduire les risques de volatilité", note d'information du rapporteur spécial sur le droit à l'alimentation, septembre 2010.

17. ABSA Bank est ainsi un « cleaning house » sur la SAFEX.

18. C. Huggins, "Commercial pressure on land in its historical perspective», Commercial Pressures on Land Research report, Rome, ILC, 2011, 46 p.

19. P.-M. Bosc et B. Losch, « Les agricultures familiales africaines face à la mondialisation », OCL, vol. 9, $\mathrm{n}^{\circ}$ 6, $2002: 402-408$.

20. S. Borras, C. Kay and E. Lahiff (eds), Market-Led Agrarian Reform: Critical Reflections on Neoliberal Land Policies and the Rural Poor, London, Routledge, 2008.

\section{RÉSUMÉS}

$\mathrm{Au}$ lendemain de l'apartheid, le secteur agricole sud-africain a connu un processus de dérégulation sans précédent, rompant ainsi avec le paternalisme étatique du régime précédent. Cette libéralisation agricole va se traduire par la montée en puissance de "macro-acteurs", entre autres des grandes banques commerciales sud-africaines, sociétés d'ingénierie agricole et fonds d'investissement, comme acteurs moteurs du secteur. Celles-ci vont progressivement renforcer leur participation au sein des filières, et particulièrement dans le segment de la production primaire agricole. De nouveaux modèles de production et de financement (ex. : « precrop contract ») apparaissent dans lesquels ces acteurs contrôlent la totalité des filières où ils sont engagés. Il apparaît ainsi une mainmise par quelques macro-acteurs (souvent non-agricoles, à structures capitalistes) sur la production agricole, considérée comme une denrée de spéculation sur les marchés financiers (Safex à Johannesbourg, marché à termes de Chicago). Si d'une part cela pose des questions sur le statut des producteurs (qui deviennent des rentiers voire des salariés des établissements, ne possédant plus leur récolte, et ne sont pas impliqués dans le processus de prise de décision, constituant ainsi un nouveau « prolétariat agricole »), d'autre part 
ces nouvelles pratiques remettent en avant les questions de souveraineté alimentaire et de régulation agricole. Ces questions sont primordiales vu qu'en l'absence d'un modèle alternatif, cette conception du développement agricole devient à présent le paradigme de référence, en Afrique du Sud mais également en Afrique. Il est en effet adopté par les agences publiques de développement (Nouveau partenariat pour le développement de l'Afrique: NEPAD, Banque africaine de développement : AfDB) et est exporté par ces « macro-acteurs » dans le cadre de leur expansion économique sur le continent.

Since the end of apartheid, the South African farming sector has been characterized by an unprecedented deregulation process, ending the state paternalism regime of the previous era. The related agricultural liberalization resulted into the engagement of "macro-actors", including South Africa's commercial banks, asset management companies and investment funds, as driving force of the sector. These actors gradually strengthened their participation within several agricultural value-chains, particularly in the primary agricultural production segment. New models of agricultural production and financing (eg "pre-crop contract") are presently mushrooming, in which these actors control the totality of the value-chain they're engaged in. These macro-actors (often non-agricultural, capitalist structures) tend to seize control over the agricultural production, considered as speculative commodities hedged on financial markets (Safex in Johannesburg, futures markets in Chicago). If these evolutions question the status of the producers (who become rent-seekers or even employees of the investing institutions and who are not possessing their harvest any more, nor are involved in the decision-making processes, constituting subsequently a new "agricultural proletariat"), they also bring to the fore issues related to food sovereignty and agricultural regulation. These issues are essential considering that in the absence of alternative development models, this conception of agricultural development presently becomes the reference paradigm, in South Africa but also on the continent. It is indeed adopted by public development agencies (NEPAD, AfDB) and is exported by these "macro-actors" within the framework of their economic expansion on the African continent.

\section{INDEX}

Index géographique : Afrique, Afrique du Sud

Keywords : Africa, agriculture, finance value-chain, hedging, South Africa, transnationalization

Mots-clés : agriculture, chaîne de valeur financière, spéculation, transnationalisation

\section{AUTEURS}

\section{ANTOINE DUCASTEL}

CIRAD et Post-Graduate School of Agriculture and Rural Development, University of Pretoria, Pretoria 0002, South Africa

antoineducastel@gmail.com

\section{WARD ANSEEUW}

CIRAD et Post-Graduate School of Agriculture and Rural Development, University of Pretoria, Pretoria 0002, South Africa.

Ward.anseeuw@up.ac.za 